\title{
Mejoría en la capacidad física después de un programa fase II de rehabilitación cardíaca, según la fracción de eyección del ventrículo izquierdo \\ Rodrigo Ibáñez, Martín Larico, Beatriz Gárate**, Max Varela*, Carolina Bascuñán*, María Teresa Carvallo* y Pía Antequera* \\ ** Enfermera Universitaria, \\ * Kinesiólogos \\ Clínica Alemana de Santiago, \\ Departamento de Cardiología, Unidad de Rehabilitación Cardíaca y Unidad Coronaria.
}

\section{Resumen:}

Antecedentes: La Fase II de un Programa de Rehabilitación Cardiaca (PRC) o post hospitalización inmediata es por definición, monitorizada, utilizando sistemas de telemetría inalámbrica, pues en esta etapa existe el mayor riesgo relacionado al ejercicio. Se sabe que los PRC mejoran la capacidad física (CF) de los pacientes y que la prueba de caminata de 6 minutos (P6) permite detectar cambios en la CF de los mismos. En Chile existen pocos estudios que evalúen los cambios en la $\mathrm{CF}$ de los pacientes según la fracción de eyección del ventrículo izquierdo (FE) estimada por ecocardiograma.

Objetivos: Evaluar los cambios en la CF después de un PRC Fase II según la FE al inicio del programa. Método: De Marzo del 2006 a Agosto del 2009 se incluyó a los pacientes que completaron PRC de nuestro centro, con 12 o 24 sesiones de ejercicios 3 veces/semana, según la clasificación de riesgo de la Asociación Americana de Rehabilitación Cardiovascular y Pulmonar (AACVPR). A todos se les realizó 2 P6 al ingreso, registrándose la mejor, y 1 P6 al final del PRC. A todos se les realizó un ecocardiograma previo al ingreso al PRC que permitió estimar la FE.

Resultados: 489 pacientes completaron el PRC Fase II. 365 Hombres (75\%), edad promedio 59,5 $\pm 14,1$ años. 114 pacientes de riesgo moderado-alto (24 sesiones). Se observó mejoría muy significativa $(\mathrm{p}<0,01)$ en la CF de la gran mayoría de los pacientes $(98,87 \%)$. El grupo con $\mathrm{FE}<30 \%$ a pesar de tener un número reducido de pacientes $(1,13 \%)$, alcanza una mejoría importante en su CF (p $<0,05)$.

Conclusiones: Con ejercicio planificado en el PRC Fase II de nuestro centro se logra una mejoría de la $\mathrm{CF}$, medido por mejoría en el test de caminata de 6 minutos, incluso en los pacientes con FE muy deprimida .

\section{Left ventricular ejection fraction and improvement of physical capacity with a cardiac rehabilitation program}

Background: During phase II cardiac rehabilitation programs (RP) or immediately after discharge requires monitorization with wireless telemetry, due to higher risk during this phase. RP are known to increase physical capacity which can be documented by the 6 min walk test (6minWT). Few studies relating improvement in physical capacity to LVEF have been reported in Chile.

Aim: to evaluate changes in physical capacity induced by a phase II RP in relation to previous LVEF.

Methods: We included patients who completed a Phase II RP from March 2006 to August 2009. 12 or 24 sessions 3 times a week were performed following recommendations from the America Association of Cardiopulmonary rehabi- litation. Two 6mWT were performed before and one after rehabilitation. For comparison, the highest measurement in pre RP was used.LVEF was determined by echocardiography before initiation of the RP.

Results: 489 patients completed the RP, $76 \%$ of them males. The mean age was $59.5 \pm 14.1$ years. 115 patients had a moderate or high risk. A highly significant $(\mathrm{p}<0.01) \mathrm{im}-$ provement in physical capacity was observed (98.9\%). The subgroup with $\mathrm{LVEF}<30 \%$, which included $1.3 \%$ of patients also had an improvement in physical capacity $(\mathrm{p}<0.05)$.

Conclusion: phase II RP achieve significant improvements in physical capacity, including patients with severely depressed LVEF. 


\section{Introducción:}

A pesar de la importante evidencia en cuanto a los beneficios de los programas de Rehabilitación Cardíaca (PRC) en los pacientes cardiópatas, son pocos los PRC que funcionan en forma completa y activamente en Latinoamérica ${ }^{1}$. La Fase II de un PRC o post hospitalización inmediata es, por definición, monitorizada, utilizando sistemas de telemetría, pues en esta etapa existe el mayor riesgo relacionado al ejercicio ${ }^{2}$. Se sabe que los pacientes en Rehabilitación Cardíaca mejoran la capacidad física ${ }^{3}$.

Para evaluar esta mejoría se han utilizado diferentes tipos de pruebas como consumo máximo de $\mathrm{O} 2$, prueba de esfuerzo y la prueba de caminata de 6 minutos; sin embargo, las primeras formas de medición son de un costo más elevado, lo que hace difícil su utilización en forma masiva como parte de un PRC. En cambio, la prueba de caminata de 6 minutos es de fácil implementación, de muy bajo costo, ha sido utilizada en diferentes grupos de pacientes para evaluar los cambios su capacidad física ${ }^{4}$, y existen valores de referencia para la población ${ }^{5,6}$. Existen numerosos estudios que han utilizado la prueba de caminata de 6 minutos en pacientes con insuficiencia cardíaca y fracción de eyección deprimida $^{7}$. En Chile no existen trabajos publicados que evalúen si la Fracción de eyección del ventrículo izquierdo (FEVI) medida por ecocardiograma al inicio del PRC es determinante para los resultados en la capacidad física de los pacientes medida por la prueba de caminata de $6 \mathrm{~min}$.

\section{Objetivos:}

Evaluar los cambios en la capacidad física de los pacientes que completan un PRC Fase II, mediante la prueba de caminata de 6 minutos. Evaluar los cambios en el resultado de la caminata de 6 minutos en relación con la fracción de eyección del ventrículo izquierdo estimada por ecocardiograma previo al inicio de la rehabilitación cardíaca.

\section{Método:}

Se realizó un estudio descriptivo prospectivo de cohorte cuya intervención es un protocolo reglado de rehabilitación física monitorizada. Desde marzo del 2006 hasta agosto del 2009, se incluyeron todos los pacientes que ingresaron al PRC Fase II, de la Clínica Alemana de Santiago y sus datos fueron registrados en una base de datos computacional específica para el efecto. Se revisaron los resultados de 489 pacientes que completaron el PRC, para efectos de esta publicación. Todos los pacientes ingresaron al programa dentro de 7 días después del egreso, luego de haber estado hospitalizados por algún evento cardiovascular. Todos los pacientes firmaron un consentimiento informado aprobado por nuestra institución que permite la utilización de los datos obtenidos durante el PRC.

Los pacientes fueron clasificados en riesgo bajo o riesgo moderado-alto, realizando 12 o 24 sesiones de ejercicios 3 veces/semana, respectivamente. Se utilizó la clasificación de riesgo de la Asociación Americana de Rehabilitación Cardiovascular y Pulmonar (AACVPR) ${ }^{2}$. En dicha clasificación, se consideran múltiples factores, dentro de los cuales la FEVI es fundamental, de manera que aquellos pacientes con $\mathrm{FEVI} \leq 50 \%$ se consideran de riesgo bajo, los que tienen FEVI entre 40 a $49 \%$ de riesgo moderado y los con $\mathrm{FEVI}<40 \%$ como de riesgo alto. En nuestro PRC todos los pacientes con FEVI $\leq 50 \%$ realizaron 24 sesiones de ejercicio. Las sesiones de ejercicio tuvieron una duración máxima de 1 hora 30 min que se distribuyeron para cada paciente en ejercicios de calentamiento, flexibilidad, caminadora y/o bicicleta, más etapa de enfriamiento. La progresión del ejercicio fue gradual e individual, aumentando primero la duración y posteriormente la intensidad del ejercicio. En las sesiones de ejercicio se utilizaron las mismas caminadoras y bicicletas Life Fitness ${ }^{\circledR}$ Classic. A todos los pacientes se les realizó un ecocardiograma 2D con Doppler previo a su ingreso al PRC y se registró la fracción FEVI. Los ecocardiogramas fueron realizados por 5 cardiólogos expertos en este método. La evaluación de la fracción de eyección se realizó usando la evaluación visual y/o el método biplano de discos (regla de Simpson modificada) según las guías internacionales descritas $^{22}$. Se utilizaron equipos de ecocardiografía GE® Vivid 7 o Dimension.

A cada paciente se le realizó dos pruebas de caminata de 6 minutos al inicio del PRC, a fin de evitar que el desconocimiento inicial de la prueba pudiese alterar los resultados ${ }^{8}$. Un kinesiólogo experto en rehabilitación cardíaca explicó a cada paciente cómo realizar el examen según las guías publicadas para la realización de esta prueba ${ }^{4}$. El mejor tiempo realizado por cada paciente se utilizó como registro de la capacidad física inicial, antes del PRC. Al término del PRC se realizó una prueba de caminata a cada paciente que se consideró la medición final de su capacidad física.

Se registraron los datos de cada paciente en cada epi- 
sodio de PRC en una base de datos creada específicamente para el efecto en programa Filemaker Pro v9.0, se analizaron los datos de dicho registro en el programa Stata v10.0.

Para describir las características demográficas de los pacientes, si hay distribución normal de la variable se expresan los resultados del cálculo como Promedio y Desviación Standard (DS) o número de casos y porcentaje respecto de la muestra según sea necesario. Para variables sin distribución normal (Fracción de eyección de VI por ej.) se expresa el resultado en Mediana y Rango Intercuartílico (RI). Se probó la distribución normal de las variables continuas con el Test de Shapiro Wilkins. Se probó la magnitud del cambio (comparación de Pre y Post PRC) del resultado de la prueba de caminata de 6 minutos con el Test de $\mathrm{T}$ de student.

\section{Resultados:}

Un grupo de 489 pacientes completaron el PRC Fase II. 365 hombres $(75 \%)$, edad promedio 59,5 $\pm 13,9$ años. 114 pacientes de riesgo moderado-alto $(23,3 \%)$. En la Tabla 1 se muestran algunas de las características de los pacientes estudiados. Destaca el alto porcentaje de hipertensión arterial (52\%) y dislipidemia (62\%). La fracción de eyección promedio de todo el grupo fue de $59 \%$ con un sesgo hacia valores mayores por lo que la mediana es 65\% con Rango Intercuartílico (RI) de 55 a $68 \%$.

Como se muestra en la Tabla 2, de los pacientes que ingresaron a nuestro PRC la mayoría presentaba enfermedad coronaria $(64 \%)$, infarto $(44 \%)$ y cirugía coronaria $(26 \%)$.

Durante las sesiones de ejercicio todos los pacientes se mantuvieron monitorizados y fueron constantemente vigilados por el equipo del PRC. En la Tabla 3 se detallan todos los eventos clínicos ocurridos durante las sesiones de rehabilitación. Se detectó arritmias supraventriculares en un $23 \%$, dentro de las cuales lo más frecuente fue la extrasistolía frecuente. Los casos con fibrilación auricular fueron escasos.

Las arritmias ventriculares fueron de poca frecuencia (8\%), con sólo 3 pacientes que presentaron taquicardia ventricular no sostenida. Los casos de hipotensión alcanzaron un $15 \%$ y en todos ellos significó algún cambio en la terapia vasodilatadora o hipotensora de los pacientes. Los pacientes que presentaron angina (4\%) fueron derivados a su cardiólogo tratante para estudio.

Como se muestra en la Tabla 4, la mayoría de los pa-

\section{Tabla 1: Descripción de la muestra en estudio}

\begin{tabular}{|c|c|c|c|}
\hline \multirow[t]{2}{*}{ Edad (prom \pm DS) en años } & \multicolumn{3}{|l|}{$59,5 \pm 13,9$} \\
\hline & & $\mathbf{N}$ & $\%$ \\
\hline \multirow[t]{2}{*}{ Sexo } & Femenino & 124 & 25 \\
\hline & Masculino & 365 & 75 \\
\hline \multirow[t]{2}{*}{ Riesgo según AACVPR } & Bajo & 376 & 76,7 \\
\hline & Moderado ñ Alto & 114 & 23,3 \\
\hline \multirow[t]{6}{*}{ Factores de riesgo CV } & Hipertensi $\mathrm{n}$ arterial & 258 & 52,7 \\
\hline & Diabetes Mellitus & 82 & 16,8 \\
\hline & Dislipidemia & 325 & 66,3 \\
\hline & Tabaquismo & 191 & 38,9 \\
\hline & Hist familiar coronaria & 198 & 40,4 \\
\hline & Obesidad & 39 & 7,9 \\
\hline \multirow[t]{2}{*}{ Fracción de Eyección VI } & Promedio & $59 \%$ & \\
\hline & Mediana (RI) & $65 \%(55-68)$ & \\
\hline
\end{tabular}

Tabla 2: Condición patológica cardiovascular previa al ingreso a PRC

\begin{tabular}{lll} 
& N & $\%$ \\
\hline Enfermedad coronaria & 313 & 64,0 \\
Infarto agudo & 217 & 44,3 \\
Muerte súbita recuperada & 5 & 1,0 \\
Cirugía coronaria & 129 & 26,3 \\
Cirugía valvular & 29 & 5,9
\end{tabular}

\section{Tabla 3: Eventos clínicos durante el desarrollo del PRC}

\begin{tabular}{lll} 
& N & $\%$ \\
\hline Total & 301 & 100 \\
Dolor torácico no anginoso & 23 & 7,6 \\
Angina & 12 & 4,0 \\
Arritmias supraventriculares & 70 & 23,2 \\
Arritmias ventriculares & 24 & 8,0 \\
Hipertensión & 23 & 7,6 \\
Hipotensión & 46 & 15,2 \\
Lipotimia & 12 & 4,0
\end{tabular}




\section{Tabla 4: Resultado de la evaluación del Test de 6 minutos según la FEVI}

\begin{tabular}{|c|c|c|c|c|c|}
\hline \multirow[t]{2}{*}{ FEVI (\%) } & \multirow[t]{2}{*}{$\%$ de la muestra } & \multicolumn{4}{|c|}{ Test de $6 \mathrm{~min}$ en $\mathrm{m} \quad$ (Prom $\pm \mathrm{DS}$ ) } \\
\hline & & Pre & Post PRC & Prom de aumento & $\mathbf{p}$ \\
\hline$<30$ & $1,1 \%$ & $431,6 \pm 198,7$ & $574 \pm 105,4$ & $142,4 \pm 127,5$ & 0,03 \\
\hline $30-39$ & $8,3 \%$ & $423,5 \pm 159,3$ & $529,8 \pm 130,0$ & $93,9 \pm 103,7$ & $<0,01$ \\
\hline $40-49$ & $8,8 \%$ & $522,7 \pm 154,0$ & $631,5 \pm 140,3$ & $112,6 \pm 79,8$ & $<0,01$ \\
\hline$\geq 50$ & $81,7 \%$ & $530,3 \pm 136,5$ & $603,8 \pm 120,2$ & $64,5 \pm 68,9$ & $<0,01$ \\
\hline Total & $100 \%$ & $521,0 \pm 142,8$ & $599,8 \pm 123,4$ & $70,8 \pm 75,3$ & $<0,01$ \\
\hline
\end{tabular}

cientes mejoró su prueba de caminata de 6 minutos con un promedio de aumento de 70,8 \pm 75,3 metros $(\mathrm{p}<0,01)$, independiente de su fracción de eyección del ventrículo izquierdo. Los pacientes con fracción de eyección de $<30 \%$ logran los mayores aumentos en la prueba de caminata de 6 minutos al final del PRC con
$142,4 \pm 127,5$ metros $(\mathrm{p}<0,05)$.

En la Tabla 5 se muestra como los pacientes clasificados en riesgo moderado o alto, que hacen 24 sesiones tienen una mejoría mayor en la prueba de caminata de 6 minutos comparado con aquellos pacientes de riesgo bajo que realizan sólo 12 sesiones de ejercicio $(\mathrm{p}<0,01)$

\begin{tabular}{|c|c|c|c|c|}
\hline \multirow{3}{*}{$\begin{array}{l}\text { Riesgo AACVPR } \\
12 \text { sesiones (riesgo bajo) }\end{array}$} & \multicolumn{4}{|c|}{$\begin{array}{l}\text { ido de la evaluación del Test } \\
\text { según el Riesgo AACVPR }\end{array}$} \\
\hline & Prom de cambio \pm DS $(m)$ & & IC & p \\
\hline & $66,6 \pm 74,3$ & 58,5 & $-75,00$ & \\
\hline \multicolumn{5}{|l|}{24 sesiones } \\
\hline (riesgo moderado-alto) & $90,4 \pm 78,4$ & 75,4 & - 105,4 & \\
\hline Total & $72,8 \pm 76,0$ & 65,5 & $-80,0$ & $<0,01$ \\
\hline
\end{tabular}

\section{Discusión:}

En la actualidad la rehabilitación cardíaca es una indicación Clase I en la mayoría de las guías clínicas para infarto con elevación del ST ${ }^{9}$, angina inestable o infarto sin elevación del $\mathrm{ST}^{10}$, angina crónica estable ${ }^{11}$, angioplastía coronaria ${ }^{12}$, cirugía coronaria ${ }^{13}$, enfermedad valvular $^{14}$, insuficiencia cardíaca ${ }^{15}$, enfermedad vascular periférica ${ }^{16}$ y prevención cardiovascular en la mujer ${ }^{17}$. La guías recomiendan un enfoque multidisciplinario que incluye no sólo ejercicio, sino también un seguimiento y evaluación nutricional, psicológica, control activo de factores de riesgo como diabetes e hipertensión, suspensión del tabaco y educación ${ }^{2,18}$ con el fin de mejorar el estilo de vida de los pacientes. Sin embargo, en países Latinoamericanos y en el Caribe donde los recursos son limitados, esta variedad de necesidades ha limitado el desarrollo de los $\mathrm{PRC}^{1}$.

Afortunadamente, se ha demostrado que no es necesario implementar un PRC tan completo para lograr beneficios sustanciales en capacidad física, calidad de vida, morbilidad y mortalidad de los pacientes coronarios, de manera que los PRC basados casi exclusivamente en terapia con ejercicio logran efectos muy favorables independientes del tipo de ejercicio aplicado o del tipo de PRC utilizado ${ }^{19,3}$. El estudio HF-ACTION ${ }^{20}$ también mostró beneficios en cuanto a capacidad física y calidad de vida en pacientes con fracción de eyección del ventrículo izquierdo $<30 \%$. En Chile son pocos los PRC que existen y la mayoría funcionan utilizando la terapia de ejercicio como el componente fundamental de la rehabilitación.

En nuestro centro el PRC tiene un fuerte enfoque educativo en cuanto a los factores de riesgo cardiovascular, mediante la orientación por parte de la enfermera del programa durante las sesiones de ejercicio y con la realización de charlas lectivas que se efectúan una vez al mes. Aquellos casos donde el aspecto psicológico o nutricional aparece como relevante son derivados a los profesionales correspondientes.

Hemos utilizado desde el comienzo del PRC la prueba de caminata de 6 minutos como herramienta evaluadora de los cambios en la capacidad física de los pacientes puesto que es de bajo costo, fácil de implementar y está al alcance de todas las personas por muy deteriorada que este su condición física. En este estudio quisimos evaluar la magnitud de los cambios que se obtenían en la capacidad física con un PRC Fase II de acuerdo a la 
fracción de eyección del ventrículo izquierdo, independiente de la patología que hubiese causado la hospitalización y el posterior ingreso al PRC.

La prueba de caminata de 6 minutos en este estudio demostró ser una herramienta muy útil para evaluar la mejoría en la capacidad física que tuvieron nuestros pacientes.

La gran mayoría de los pacientes logró una mejoría significativa de su capacidad física independiente de su fracción de eyección $(98,87 \%)$. El grupo con FE $<30 \%$ a pesar de tener un número reducido de pacientes $(1,13 \%)$, alcanza una mejoría importante en su capacidad física $(\mathrm{p}<0,05)$ con un promedio de aumento de 142 metros, muy superior a lo reportado en recientes estudios $^{20}$ y comparable a lo descrito para la terapia farmacológica óptima ${ }^{21}$.Tal vez esta diferencia se deba a que todos estos pacientes realizaron 24 sesiones de ejercicios supervisados en nuestro gimnasio, vigilados con telemetría, lo que permitió generar con seguridad avances más rápidos en su capacidad funcional, de hecho es el grupo de riesgo moderado-alto (24 sesiones) el que tiene la mayor mejoría en la prueba de caminata de 6 min respecto al grupo de riesgo bajo (12 sesiones) $(\mathrm{p}<0,01)$.

En esta experiencia se detectaron 301 eventos clínicos, de los cuales casi el $30 \%$ fueron algún tipo de arritmia. A pesar que la minoría fueron arritmias que requirieron un cambio de conducta significativo (fibrilación auricular, flutter auricular o taquicardia ventricular) la telemetría y la supervisión por el personal del PRC fue fundamental para la adecuada detección y manejo de estos eventos.

\section{Conclusiones:}

Se confirma que la prueba de caminata de 6 minutos es un método factible y fiable para medir la capacidad física de los pacientes en el contexto de un PRC. Con ejercicio supervisado y monitorizado, el PRC logra una mejoría de la CF incluso en los pacientes con FE muy deprimida y en una magnitud que puede ser superior a lo logrado por otras terapias.

En la rehabilitación física de los pacientes con indicación de completar un PRC suceden eventos clínicos que requieren de monitorización tanto clínica como de telemetría electrocardiográfica para su precisa identificación, prevención y tratamiento.

\section{Referencias:}

1. KORENFELD Y, MENDOZA-BASTIDAS C, SAAVEDRAL, MONTERO-GÓMEZ A, PEREZ-TERZIC C, THOMAS RJ, et al. Current status of cardiac rehabilitation in Latin America and the Caribbean. Am Heart J 2009; 58: 480-7.

2. American Association of Cardiovascular and Pulmonary Rehabilitation. Guidelines for Cardiac Rehabilitation and Secondary Prevention Programs. Champaign, IL: Human Kinetics, 2004.

3. WENGER NK. Current Status of Cardiac Rehabilitation. J Am Coll Cardiol 2008; 51: 1619-31.

4. ATS statement: guidelines for the six-minute walk test. Am J Respir Crit Care Med 2002; 166: 111-117

5. TERAMOTO S, OHGA E, ISHII T, YAMAGUCHI Y, YAMAMOTO H, MASTUSE T. Reference value of six-minute walking distance in healthy middle-aged and older subjects. Eur Respir J 2000; 15: 1132-1133.

6. TROOSTERS T, GOSSELINK R, DECRAMER M. Six minute walking distance in healthy elderly subjects. Eur Respir J 1999; 14: $270-274$

7. BALASHOV K, FELDMAN DE, SAVARD S, HOUDE $\mathrm{S}$, FRENETTE M, DUCHARME A, et al. Percent Predicted Value for the 6-Minute Walk Test: Using Norm-Referenced Equations to Characterize Severity in Persons With CHF. J Card Fail. 2008; 14: 75-81.

8. WU G, SANDERSON B, BITTNER V. The 6-minute walk test: How important is the learning effect? Am Heart J 2003; 146: 12933.

9. ANTMAN EM, ANBE DT, ARMSTRONG PW, BATES ER, GREEN LA, HAND M, et al. ACC/AHA guidelines for the management of patients with ST-elevation myocardial infarction: executive summary: a report of the American College of Cardiology/American Heart Association Task Force on Practice Guidelines (Committee to Revise the 1999 Guidelines on the Management of Patients with Acute Myocardial Infarction). J Am Coll Cardiol 2004; 44: 671-719.

10. ANDERSON JL, ADAMS CD, ANTMAN EM, BRIDGES CR, CALIFF RM, CASEY DE JR, et al. ACC/AHA 2007 guidelines for the management of patients with unstable angina/non STelevation myocardial infarction: a report of the American College of Cardiology/American Heart Association Task Force on Practice Guidelines (Writing Committee to Revise the 2002 Guidelines for the Management of Patients With Unstable Angina/Non-STElevation Myocardial Infarction): developed in collaboration with the American College of Emergency Physicians, American College of Physicians, Society for Academic Emergency Medicine, Society for Cardiovascular Angiography and Interventions, 
and Society of Thoracic Surgeons. J Am Coll Cardiol 2007; 50: e1-157.

11. GIBBONS RJ, ABRAMS J, CHATTERJEE K, DALEY J, DEEDWANIA PC, DOUGLAS JS, et al. ACC/AHA 2002 guideline update for the management of patients with chronic stable angina summary article: a report of the American College of Cardiology/American Heart Association Task Force on Practice Guidelines (Committee on the Management of Patients with Chronic Stable Angina). J Am Coll Cardiol 2003; 14: 159-68.

12. SMITH SC JR, FELDMAN TE, HIRSHFELD JW JR, JACOBS AK, KERN MJ, KING SB 3RD, et al. ACC/AHA/SCAI 2005 guideline update for percutaneous coronary intervention summary article: a report of the American College of Cardiology/ American Heart Association Task Force on Practice Guidelines (ACC/AHA/SCAI Writing Committee to Update the 2001 Guidelines for Percutaneous Coronary Intervention). J Am Coll Cardiol 2006; 47: 216-35.

13. EAGLE KA, GUYTON RA, DAVIDOFF R, EDWARDS FH, EWY GA, GARDNER TJ, et al. ACC/AHA 2004 guideline update for coronary artery bypass graft surgery: summary article: a report of the American College of Cardiology/American Heart Association Task Force on Practice Guidelines (Committee to Update the 1999 Guidelines for Coronary Artery Bypass Graft Surgery). J Am Coll Cardiol 2004; 44: e213-310

14. BONOW RO, CARABELLO BA, CHATTERJEE K, DE LEON AC JR, FAXON DP, FREED MD, et al. ACC/AHA 2006 guidelines for the management of patients with valvular heart disease: a report of the American College of Cardiology/American Heart Association Task Force on Practice Guidelines (Writing Committee to Develop Guidelines for the Management of Patients with Valvular Heart Disease). J Am Coll Cardiol 2006; 114: e1-148.

15. HUNT SA, ABRAHAM WT, CHIN MH, FELDMAN A, FRANCIS G, GANIATS T, et al. ACC/AHA 2005 guideline update for the diagnosis and management of chronic heart failure in the adult-summary article: a report of the American College of Cardiology/American Heart Association Task Force on Practice Guidelines (Writing Committee to Update the 2001 Guidelines for the Evaluation and Management of Heart Failure). J Am Coll Cardiol 2005; 46: 1116-43.

16. HIRSCH AT, HASKAL ZJ, HERTZER NR, BAKAL CW, CREAGER MA, HALPERIN JL, et al. ACC/AHA guidelines for the management of patients with peripheral arterial disease (lower extremity, renal, mesenteric, and abdominal aortic): exe- cutive summary: a collaborative report from the American Association for Vascular Surgery/Society for Vascular Surgery, Society for Cardiovascular Angiography and Interventions, Society for Vascular Medicine and Biology, Society of Interventional Radiology, and the ACC/AHA Task Force on Practice Guidelines (Writing Committee to Develop Guidelines for the Management of Patients With Peripheral Arterial Disease). J Am Coll Cardiol 2006; 47: 1239-312.

17. MOSCA L, BANKA CL, BENJAMIN EJ, BERRA K, BUSHNELLC, DOLOR RJ, et al., for the Expert Panel/Writing Group. Evidence-based guidelines for cardiovascular disease prevention in women: 2007 update. Circulation 2007; 115: 1481-501.

18. BALADY GJ, WILLIAMS MA, ADES PA, BITTNER V, COMOSS P, FOODY JM, et al. Core components of cardiac rehabilitation/secondary prevention programs: 2007 update. A scientific statement from the American Heart Association Exercise, Cardiac Rehabilitation, and Prevention Committee, the Council on Clinical Cardiology; the Councils on Cardiovascular Nursing, Epidemiology and Prevention, and Nutrition, Physical Activity, and Metabolism; and the American Association of Cardiovascular and Pulmonary Rehabilitation. Circulation 2007; 115: 2675-82.

19. TAYLOR RS, BROWN A, EBRAHIM S, JOLLIFFE J, NOORANI H, REES K, et al. Exercise-based rehabilitation for patients with coronary heart disease: systematic review and metaanalysis of randomized controlled trials. Am J Med 2004; 116: 682-92.

20. O'CONNOR CM, WHELLAN DJ, LEE KL, KETEYIAN SJ, COOPER LS, ELLIS SJ, et al. Efficacy and Safety of Exercise Training in Patients With Chronic Heart Failure. HF-ACTION Randomized Controlled Trial. JAMA. 2009; 301: 1439-1450.

21. GUALENIA,D'ALOIAA, GENTILINIA,PAGANI M,GIORDANO A, FAGGIANO P. Effects of maximally tolerated oral therapy on the six-minute walking test in patients with chronic congestive heart failure secondary to either ischemic or idiopathic dilated cardiomyopathy. Am J Cardiol.1998; 81: 1370-72.

22. LANG RM, BIERIG M, DEVEREUX RB, FLACHSKAMPF FA, FOSTER E, PELLIKKA PA, et al. Recommendations for Chamber Quantification: A Report from the American Society of Echocardiography's Guidelines and Standards Committee and the Chamber Quantification Writing Group, Developed in Conjunction with the European Association of Echocardiography, a Branch of the European Society of Cardiology. J Am Soc Echocardiogr 2005; 18: 1440-1463. 